\title{
Recognition of emission from the wood based products waste combustion using differential ion mobility spectrometry
}

\author{
Monika Maciejewska ${ }^{1, *}$, Andrzej Szczurek ${ }^{1}$, and Żaneta Zajiczek ${ }^{1}$ \\ ${ }^{1}$ Faculty of Environmental Engineering, Wroclaw University of Science and Technology, \\ Wybrzeże Wyspiańskiego 27, 50-370 Wrocław, Poland
}

\begin{abstract}
This work was focussed on the recognition of the emission of volatile compounds resulting from the combustion of engineered wood products waste. This kind of waste is broadly used for heating purposes in an unauthorised way, giving rise to unorganised emissions. The recognition of such events is very difficult due to the complexity of the produced gas mixture. We proposed to apply differential ion mobility spectrometry (DMS). This is a promising technique in terms of complex gas mixtures measurements. The recognition was based on the measurements of ambient air in the vicinity of the emission source and classification. The ensemble of classification trees was chosen as the classier. The obtained results showed that volatile compounds resulting from the combustion of wood based boards waste produced the distinctive DMS spectra, which could be used as the basis for the effective recognition. We achieved almost $100 \%$ successful recognition of: 1) ambient air which contained volatile compounds resulting from OSB board waste combustion, 2) ambient air which contained volatile compounds resulting from MDF board waste combustion, and 3) ambient air, which was did not contain volatile compounds of this kind. The presented results have a considerable practical value. The DMS spectrometer was successfully applied to recognize wood-based boards waste combustion in field conditions.
\end{abstract}

\section{Introduction}

Polish production and processing of engineered wood products is one of the most modern industries of this type in Europe and throughout the world [1]. Numerous kinds of boards are manufactured from wood chips, wood flakes, sawmill shavings etc., which are mixed with suitable binders. The binders are glues based on various kinds of resins, paraffin wax and water. The additives constitute up to $10 \%$ of the overall mass. The mixture is pressed and extruded giving rise to composite materials. The most popular boards are the particle board, also known as chipboard or low density fibre board (LDF), medium density fibre board (MDF) and oriented strand board (OSB). All of them are commercially available in

\footnotetext{
*Corresponding author: monika.maciejewska@pwr.edu.pl
} 
large number of varieties. The major consumer of boards is furniture industry, which uses about $70 \%$ of the production. The rest goes to multiple other branches of wood processing industry.

Manufacturing and processing of engineered wood products involves generation of large volume of waste [2]. In this particular case the waste management problem has not been successfully solved. The waste has high calorific value, e.g. $16.6 \mathrm{MJ} / \mathrm{kg}$ for OSB, 18.2 $\mathrm{MJ} / \mathrm{kg}$ for MDF, which makes it useful for heat production purposes. However, binders, added during the manufacturing process influence the combustion process and significantly change the composition of the flue gas, as compared to raw wood. In order to eliminate the risk of noxious emissions, the combustion process has to be run at the defined conditions, in the dedicated installations, which are fitted with the appropriate emission control equipment $[3,4]$. Household boilers do not fulfil these requirements. Unfortunately, cheap price, broad availability and easy access to engineered wood products waste encourage its use as a fuel in an unauthorised way. This kind of activity poses an environmental threat, which is particularly serious in case of the unorganised emission. For this reason there are needed methods of detection of this kind of events, at site.

Reaching this goal is not easy in practice. Due to the complex composition of wood based products, the profiles of emission from their combustion are very complex. In general, the analytical devices, which are capable of performing extensive qualitative and quantitative analysis of gas samples are prepared to operate in laboratory conditions. Environmental engineering faces an urgent need of developing a new generation of measurement devices. They are expected to operate in field conditions and provide comprehensive information, especially in cases when the emitted gases are complex in terms of their chemical composition. Moreover, they shall be featured by the low price and the simplicity of operation.

The aim of this work was to present the results of the study, in which a measurement device, based on differential ion mobility spectrometry (DMS) was applied to detect and recognize the mixture of volatile compounds resulting from the combustion of waste of engineered wood products in a stove which was not dedicated for this purpose. The study is a part of research oriented at the detection of unwanted, uncontrolled emissions based on the response of the measuring device rather than by determining a marker. The proposed approach is highly innovative and practical at the same time.

\section{Experimental part}

\subsection{Materials}

Two types of wood composite products were considered in the study as the exemplary sources of wood engineered products waste: medium-density fibreboard (MDF) and oriented strand board (OSB). They are popular and commonly used kinds of board.

Medium-density fibreboard is made by breaking down hardwood or softwood residuals into wood fibres, combining it with wax and a resin binder. After that, panels are formed by applying high temperature and pressure. MDF is typically made up of $82 \%$ wood fibre, $9 \%$ urea-formaldehyde resin glue, $8 \%$ water and $1 \%$ paraffin wax [5]. MDF is generally denser and stronger than plywood and particle board. Their large scale production is located mainly in China and in Europe. The MDF used in the experiment was manufactured by Kronospan Mielec Sp. Zoo [6], see Fig. 1.

Oriented strand board (OSB), also known as flakeboard, is formed by adding adhesives and then compressing layers of wood strands (flakes) in specific orientations. OSB may have a rough and variegated surface with the individual wood strips of several centimetres 
long, lying unevenly across each other. Oriented strand board is typically made of $95 \%$ wooden strips compressed and bonded together with 5\% wax, resin wax and synthetic resin adhesives. OSBs come in a variety of kinds. The adhesive resins types used include: urea-formaldehyde (OSB type 1, non-structural, non-waterproof); isocyanate based glue (or PMDI poly-methylene diphenyl diisocyanate based) in inner regions with melamine-urea-formaldehyde or phenol formaldehyde resin glues at surface (OSB type 2, structural, water resistant on face); phenol formaldehyde resin throughout (OSB types 3 and 4, structural, for use in damp and outside environments) [7]. OSB is similar to particle board. The OSB type 3, manufactured by Kronospan Mielec Sp. Zoo [8], was used in the experiment, see Fig. 1.
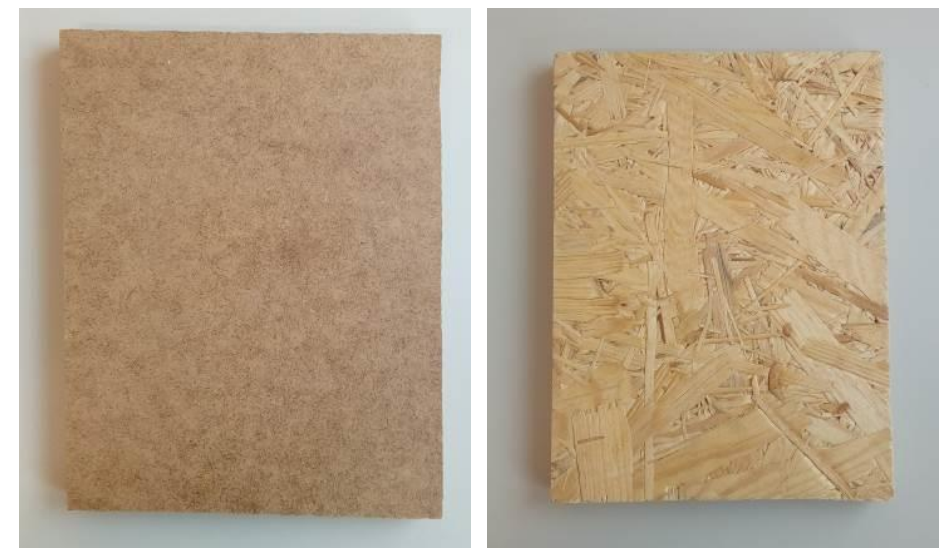

Fig. 1. MDF (left) and OSB (right) boards which were examined in the study.

\subsection{Experimental setup}

The experimental setup consisted of a stove and the measurement setup.

The popular stove, type Ackerman PW $2.5 \mathrm{KW}$ was chosen to simulate the unauthorised combustion process of engineered wood product waste. Pieces of MDF and OSB boards were combusted in the stove individually. The combustion rate was adjusted by the rate of stove feeding. We added approximately 1 piece $(20 \mathrm{~cm} \times 15 \mathrm{~cm})$ every $10 \mathrm{~min}$. The flue gas was emitted through a vertical chimney, The release point was at the height of $3 \mathrm{~m}$ above the ground. By choosing a small stove and not very intensive combustion we achieved the desired test conditions. The emission was relatively small, compared with any realistic cases of emission from board waste combustion for heating purposes. Hence, the proposed measurement approach was tested in the unfavourable conditions of small emission.

The stove was located in a self-standing outbuilding with no other buildings around it in the radius of $10 \mathrm{~m}$. The terrain was flat.

The measurement set-up consisted of differential ion mobility spectrometer and the polyethylene tubing. The setup allowed to perform measurements around the emission source. The ambient air was sampled at four sampling points around emission source (see Fig. 2) at the height of about $1.5 \mathrm{~m}$ above the ground. Tubes were used for delivering air samples from the sampling points to the benchtop spectrometer. Points were served in a cyclic manner, one sampling point a time. Each time, the air was sampled from a particular sampling point, at least three ion mobility spectra were recorded. Between-points switching was done with a time step of 2 min approximately. 


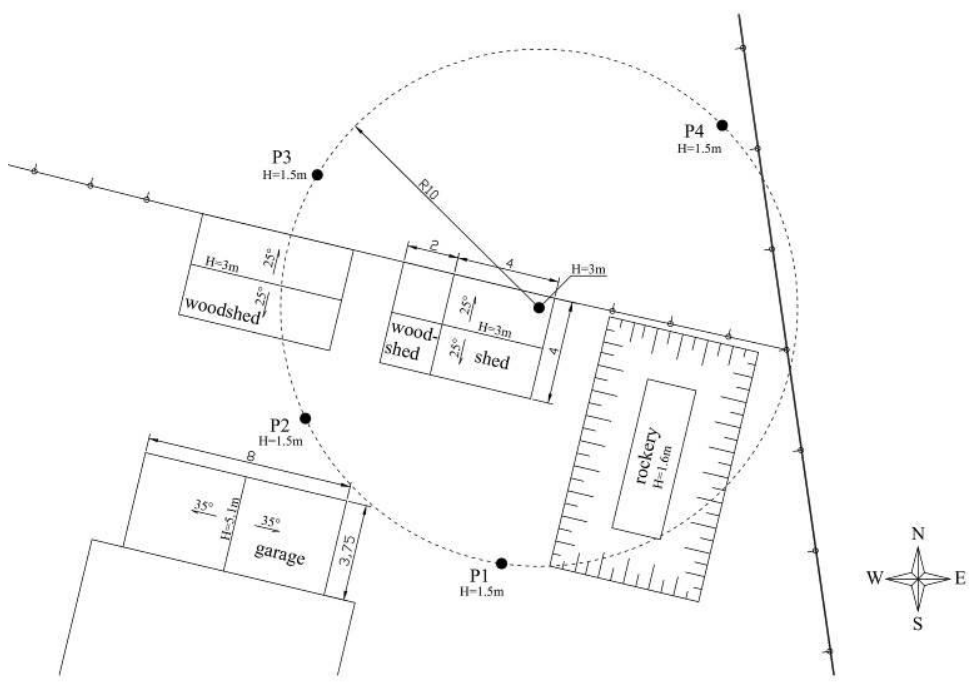

Fig. 2. The situation plan.

The experiment related to the detection, in ambient air, of volatiles resulting from MDF board waste combustion was run for three days. Each day, ambient air was monitored for about two hours during the combustion process. Additionally, the measurements of the ambient air baseline were done each day, at least two hours before initiating the combustion process. The same approach was applied in the experiment related to the detection of volatiles resulting from OSB board combustion. In total, there were collected 600 differential ion mobility spectra of ambient air during MDF combustion, 630 spectra of ambient air during OSB board combustion and 80 baseline spectra of ambient air, i.e. when no combustion took place for at least several hours in advance.

\subsection{Differential ion mobility spectrometer}

Differential ion mobility spectrometry is a promising analytical technique, which allows for the qualitative and quantitative characterization of gas mixtures [9]. It expands on the ion mobility spectrometry, which has already found its commercial applications.

The DMS technique utilises differences between the mobility of ions in weak and strong electric field. The analysed gas sample is ionized and the obtained ions are passed through the special chamber with the carrier gas. In the chamber, the ions are intermittently exposed to the weak and strong electric field. The frequency and amplitude of the variable electric field are high. Due to the asymmetry of the voltage waveform, which is responsible for field generation (separation voltage, SV), the bundle of ions in the chamber is tilted. For tilt correction the compensation voltage $(\mathrm{CV})$ is applied. As the tilt mostly depends on the kind of ion, different ions arrive to the collection electrodes at different combinations of separation voltage and compensation voltage. This is the basis for their qualitative determination. On the other hand, the intensity of the ionic current may be correlated with the number of ions of the particular kind in the gas sample.

The prototype DMS spectrometer applied in the study was schematically shown in Fig. 3. Its detailed description was presented elsewhere [10]. 


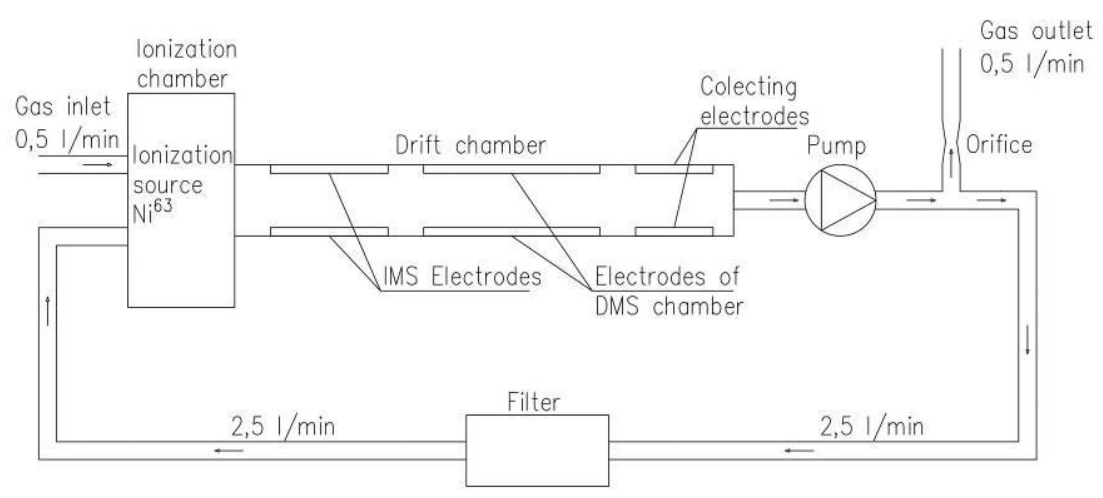

Fig. 3. Schematic view of differential ion mobility spectrometer which was applied in the study.

The result of the measurement done with differential ion mobility spectrometer is the two dimensional spectrum. It presents an ionic current as a function of separation and compensation voltages. The spectrum contains the information about all volatile compounds present in gas sample, which are susceptible to ionization, by the ionization source applied in the instrument, and the produced ions are capable of reaching the collecting electrode in the predefined ranges of compensation and separation voltages. The differential ion mobility spectrum raises and interest as a source of information about complex gas mixtures. It is particularly interesting, when combined with the techniques of multivariate data processing, for example with classification techniques [11].

\section{Classification}

The objective of the classification was to distinguish the differential ion mobility spectra of: 1) ambient air which contained the volatile compounds resulting from the combustion of MDF board waste, 2) ambient air which contained the volatile compounds resulting from the combustion of OSB board waste, and 3) ambient air which did not contain the compounds of such origin. Three respective classes were defined, the first including 600 spectra, the second including 630 spectra and the third including 80 spectra.

The classification was based on the entire DMS spectrum. Let's define the individual spectrum as the matrix $\mathbf{R}=\left[\mathrm{r}_{\mathrm{i}, \mathrm{j}}\right]$, where $\mathrm{r}_{\mathrm{i}, \mathrm{j}}$ is the response of the collecting electrode (ionic current) recorded for the particular combination of separation voltage $S V_{i}, i=1 \ldots n$, and $C V_{j}$, $\mathrm{j}=1 \ldots \mathrm{m}$. The input vector of the classifier was the vector, which included all elements of matrix R, arranged in the predefined order. The output of the classifier was the label of the respective class.

An ensemble of classification trees was chosen as the classification model for the examined problem. Our choice was inspired by the successful use of ensembles in face recognition based on digital images [12]. The main principle behind an ensemble is that a group of weak learners come together to form a strong learner. For example, several combined decision trees produce better predictive performance than utilizing a single decision tree. The ensemble response is predicted by aggregating predictions from its weak learners, according to the predefined rule.

We developed and tested the classifier ensemble which consisted of 100 classification trees. It was trained using AdaBoost.M2 algorithm, which is an ensamble aggregation method designed for multiclass problems with weak base classifiers. There were allowed maximum 5 splits in one tree. 
The performance of the classification model was evaluated using ten-fold cross-validation procedure. The results of model validation were presented in the form of confusion matrix, which allowed for their detailed examination. The model will be optimised in our future work.

\section{Results and discussion}

Fig. 4 shows the median of differential ion mobility spectra of: 1) ambient air which contained volatile compounds resulting from OSB board waste combustion, 2) ambient air which contained volatile compounds resulting from MDF board waste combustion, and 3) ambient air, which was did not contain the volatile compounds of this kind. The displayed spectra refer to positive ions. Negative ions spectra were not applicable to support the considered application.
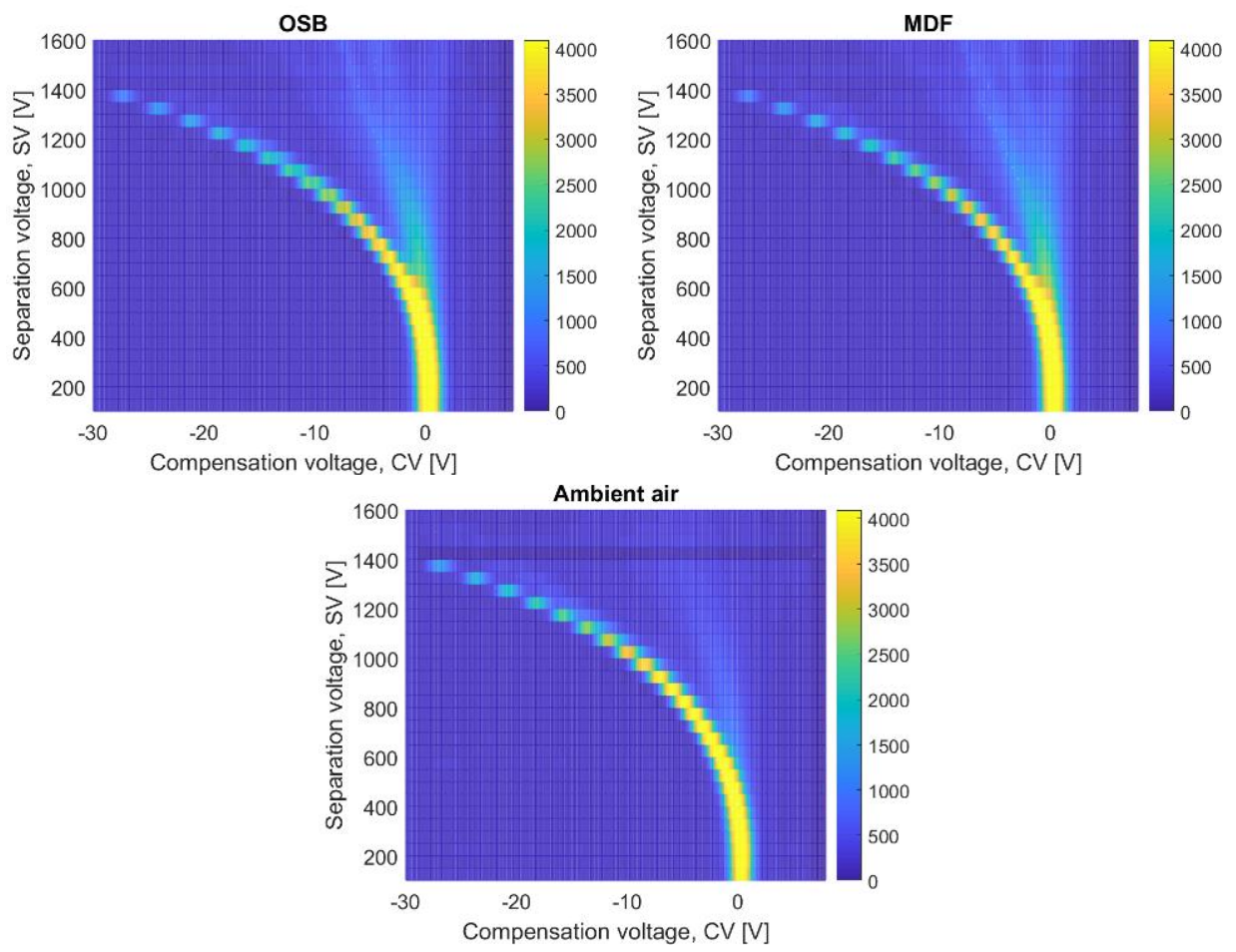

Fig. 4. Medians of all DMS spectra (positive ions) for: ambient air nearby the emission source during OSB waste combustion (top left), ambient air nearby the emission source during the MDF waste combustion (top right), and baseline ambient air (bottom). Spectra were recorded in the vicinity of the emission source.

The spectra compared in Fig. 4, are complex. In each displayed spectrum, the recorded ionic current varied as a function of the separation voltage and the compensation voltage. Spectra may be considered as patterns, which represent the various kinds of the measured gases. Differences between spectra give rise to gases recognition. As shown in Fig. 4, the most distinctive region of the spectrum was delimited by the following tops of the triangle $(\mathrm{SV}=1600 \mathrm{~V}, \mathrm{CV}=-15 \mathrm{~V}),(\mathrm{SV}=1600 \mathrm{~V}, \mathrm{CV}=-5 \mathrm{~V})$ and $(\mathrm{SV}=400 \mathrm{~V}, \mathrm{CV}=0 \mathrm{~V})$. In particular, in this region of spectrum the ionic current was small for baseline air. In case of 
air affected by the volatiles resulting from waste combustion, the ionic current was much higher in the mentioned region. Based on visual inspection, the spectra associated with the combustion of MDF and OSB board waste were not so notably different. But, the actual differences were sufficient for the classification model.

Table 1 presents the confusion matrix for the classification of DMS spectra of ambient air nearby the emission source during the combustion of medium-density fibreboard (MDF), oriented strand board (OSB) and when none of them was combusted. The presented results refer to ten-fold cross validation procedure applied to examine the performance of the classification model.

Table 1. Confusion matrix for the classification of DMS spectra of ambient air nearby the emission source during combustion of medium-density fibreboard (MDF), oriented strand board (OSB) and none of them. The presented results refer to ten-fold cross validation.

\begin{tabular}{|c|c|c|c|c|}
\cline { 3 - 5 } \multicolumn{2}{c|}{} & \multicolumn{3}{c|}{ PREDICTED } \\
\cline { 3 - 6 } \multicolumn{2}{c|}{} & MDF & OSB & None \\
\cline { 2 - 5 } \multirow{3}{*}{ TRUE } & OSB & $599(99.83 \%)$ & 0 & $1(0.17 \%)$ \\
\cline { 2 - 5 } & None & $1(1.27 \%)$ & 0 & 0 \\
\hline
\end{tabular}

As shown in Table 1 the DMS spectra of ambient air nearby the emission source was an effective source of information about the combusted material. In particular, the spectra allowed for the discrimination of the occurrence of MDF board waste combustion from OSB board waste combustion. This task was solved with $100 \%$ efficiency. Based on Table 1 none spectrum of air containing volatiles released during MDF combustion was confused with the spectrum referring to OSB combustion. The performed analysis showed additionally that the occurrence of the combustion process was very well discriminated from its lack, based on DMS spectra. None mistake was made in case of OSB board. The volatiles released during its combustion appears to have a very specific DMS spectrum. In case of MDF board there were noted two misclassifications. One spectrum of air including volatiles from MDS combustion was mistakenly taken for the spectrum of air not affected by the those volatiles $(0.17 \%$ misclassification rate). Also, one spectrum of air not affected by the volatiles was mistakenly classified as the spectrum associated with MDS combustion ( $1.27 \%$ misclassification rate). These misclassification rates are negligible. Moreover, it is worth noticing that the probability of detecting emission when it has not occurred is greater as compared with probability of claiming lack of emission while the emission actually occurred. This kind of bias is favoured for practical reasons.

\section{Conclusions}

This work was focussed on the recognition of the emission of volatile compounds resulting from the combustion of engineered wood products waste. This kind of waste is broadly used for heating purposes in an unauthorised manner, giving rise to the unorganised emissions. The recognition of such occurrences is very difficult due to the complexity of the gas mixture.

Differential ion mobility was considered as the promising measurement technique in this kind of applications. The recognition was based on the measurements of ambient air in the vicinity of the emission source and classification. The DMS spectra were utilised as the basis for the classification. The ensemble of classification trees was chosen as the classier.

The obtained results showed that the volatile compounds resulting from the combustion of wood-based boards waste were suitable for gas phase chemical ionization and produced 
a complex response in terms of DMS spectrum. The spectra were distinctive and could be used the basis for effective recognition.

We demonstrated almost $100 \%$ successful recognition of: 1) ambient air which contained volatile compounds resulting from OSB board waste combustion, 2) ambient air which contained volatile compounds resulting from MDF board waste combustion, and 3 ) ambient air, which was did not contain the volatile compounds of this kind.

The presented results have a considerable practical value. They were based on field measurements which were done in multiple measurement points, on different days and at different times of the day. The DMS spectrometer was successfully applied to detect wood-based boards waste combustion in real conditions.

\section{References}

1. http://www.biznesmeblowy.pl/produkcja_mebli/116/produkcja_tarcicy_i_plyt_w_i_pol roczu_2017_roku,12225.html

2. M. Kajda-Szcześniak, Archiwum Gospodarki Odpadami i Ochrony Środowiska, 15 (2013)

3. Rozporządzenie Ministra Rozwoju Dz.U. 2016 poz. 108

4. Rozporządzenie Ministra Środowiska Dz.U. 2018 poz. 680

5. B. J. A. Youngquist, A. M. Krzysik, Lignocellulosic Composites (in: G. Richard Ed. Cellulosic polymers, blends and composites, New York, Hanser Publishers, 1994)

6. Atest higieniczny HK/B/0812/01/2015

7. T. Oldhand, "Types of Glue Used in OSB", sciencing.com 2017

8. Deklaracja właściwości użytkowych Nr: ST_OSB/3_CPR_2015

9. G. A. Eiceman, Z. Karkas, H. H. Hill, Ion mobility spectrometry ( $3^{\text {rd }}$ edition, CRP Taylor \& Francis Group, Boca Raton, 2014)

10. A. Szczurek, M. Maciejewska, Ż. Zajiczek, Eko-Dok (2019) (to be published)

11. A. Szczurek, M. Maziejuk, M. Maciejewska, T. Pietrucha, T. Sikora, Sensors and Actuators B: Chemical, 240 (2017)

12. J. Lu, K. N. Plataniotis, A. N. Venetsanopoulos, S. Z. Li, IEEE Transactions on Neural Networks 17, 1 (2006) 